\title{
Intervenção Coronária Percutânea após Trombólise no Infarto Agudo do Miocárdio: Para Quem e Quando?
}

\author{
Ver artigo relacionado \\ na página 373
}

\author{
Alexandre Schaan de Quadros ${ }^{1}$
}

A intervenção coronária percutânea (ICP) primária é o método preferencial para reperfusão no infarto agudo do miocárdio com supradesnivelamento do segmento ST (IAMCST). No entanto, muitos pacientes se apresentam em centros que não dispõem desse método. Quando o tempo de transferência até um hospital habilitado a realizar ICP primária na fase aguda do IAM é muito longo, a terapia fibrinolítica é utilizada como método de reperfusão inicial. Em caso de insucesso do trombolítico para reperfundir a artéria coronária relacionada ao infarto, os pacientes devem ser transferidos para um centro com capacidade de ICP primária o mais breve possível (ICP primária de resgate). Se houve sucesso da trombólise, o paciente pode ainda ser submetido a ICP eletiva nas primeiras 24 horas. Caso não exista essa possibilidade, deve ser estratificado para o risco de novos eventos cardiovasculares, sendo utilizados testes não-invasivos para avaliação de isquemia miocárdica e cineangiocoronariografia. Nesse contexto, as indicações para estratificação invasiva ou não e as indicações de recanalização da artéria relacionada ao infarto têm sido objeto de atenção e intensa controvérsia.

Nesta edição, Lima et al. ${ }^{1}$ relatam os dados provenientes do Registro Madre Teresa de Belo Horizonte (MG), que avaliou 303 pacientes com diagnóstico de IAM submetidos a reperfusão química e transferidos para esse centro. $O$ tempo médio de realização da ICP foi tardio (5 dias após o IAM), e três quartos dos pacientes apresentavam fluxo TIMI 3 antes dos procedimentos. As taxas de sucesso angiográfico e de sucesso clínico foram de 95\% e $87 \%$, respectivamente, e a taxa de mortalidade hospitalar foi de apenas 3\%. Esses dados são bastante encorajadores, e atestam para a experiência dos operadores e a excelência do centro em questão, mas devemos considerar os critérios de seleção empregados no estudo antes da extrapolação desses dados. Especificamente, foram incluídos pacientes ao longo de 7 anos e meio de estudo, sendo excluídos aqueles com ICP de resgate e com a artéria relacionada ao infarto ocluída antes do procedimento. Outro aspecto importante é que informações referentes à isquemia induzida e presença de angina do peito não foram relatadas nesse estudo.

Recentemente, foram publicadas as últimas diretrizes conjuntas do American College of Cardiology/ American Heart Association/Society for Cardiovascular Angiography and Interventions para intervenções coronárias percutâneas, e que constituem o documento mais atualizado sobre o tema. ${ }^{2}$ As recomendações atuais para ICP após trombólise no IAM são as seguintes: 1) ICP de resgate após falha aguda do trombolítico (classe Ila, nível de evidência B); 2) ICP eletiva, 3-24 horas após trombólise bem-sucedida (classe Ila, nível de evidência B); e 3) ICP após o primeiro dia do IAM naqueles com isquemia residual ou angina (classe Ila, nível de evidência B). A ICP em artérias patentes e com estenose grave mas sem isquemia ou angina residuais é considerada como classe Ilb, nível de evidência B. Finalmente, ICP de uma artéria ocluída após as primeiras 24 horas em pacientes assintomáticos e estáveis é considerada como classe III, nível de evidência B.

Como não dispomos de informações sobre a presença de isquemia residual no estudo de Lima et al. ${ }^{1}$, não é possível caracterizar o grau de recomendação desses pacientes, que poderia ser Ila ou Ilb. É importante ressaltar que a recomendação Ilb não significa que o procedimento não deva ser realizado, mas apenas que provavelmente não seja recomendado na maioria dos pacientes. Em virtude do longo tempo de inclusão do estudo citado, e da ausência da descrição detalhada da população de pacientes pós-trombólise da qual a amostra foi selecionada, é possível que esse estudo tenha incluído um subgrupo de pacientes com condição clínica favorável para ICP tardia em artérias com estenose grave, mesmo sem isquemia miocárdica residual demonstrada. Esse aspecto deve também ser

\footnotetext{
1 Instituto de Cardiologia do Rio Grande do Sul - Fundação Universitária de Cardiologia (IC-FUC) - Porto Alegre, RS, Brasil Correspondência: Alexandre Schaan de Quadros. Av. Princesa Isabel, 370 - Porto Alegre, RS, Brasil - CEP 90620-001 E-mail: alesq@terra.com.br

Recebido em: 13/12/2011 • Aceito em: 14/12/2011
} 
considerado ao extrapolar os resultados do estudo para outras populações.

Finalmente, para conciliar e colocar em perspectiva na realidade brasileira os resultados do estudo de Lima et al. ${ }^{1}$ e as recomendações da diretriz americana, é importante considerar dois cenários distintos. Atualmente, os pacientes com planos de saúde privados no Brasil em geral têm acesso adequado a exames e tratamentos preconizados pelas diretrizes. Por outro lado, aqueles pacientes atendidos pelo Sistema Único de Saúde (SUS) sofrem com restrições de procedimentos, tanto em relação à possibilidade de sua realização quanto a seu emprego em tempo hábil para o melhor tratamento de sua afecção. No caso específico da ICP após IAM, existe maior facilidade na realização da cineangiocoronariografia e da ICP que na realização de testes diagnósticos não-invasivos para avaliação de isquemia residual e viabilidade miocárdica, que muitas vezes não são disponíveis.

É fundamental que essas distorções do sistema de saúde sejam adequadamente avaliadas e resolvidas, para que todos os pacientes possam receber o melhor tratamento possível após o IAM. Nesse contexto, um importante passo foi dado recentemente, com o lançamento dos programas nacionais do Ministério da Saúde para Protocolo de Trombólise no SUS e para Estímulo das Unidades Coronárias. O programa de trombólise prevê a inclusão da alteplase, da tenecteplase e do clopidogrel, além de dosagem de troponina, na tabela de pagamentos do SUS. Essa iniciativa irá qualificar o atendimento ao infarto no setor público, e deverá se traduzir em diminuição das taxas de mortalidade e de complicações relacionadas a essa afecção de elevada prevalência.

\section{CONFLITO DE INTERESSES}

O autor declara não haver conflito de interesses relacionado a este manuscrito.

\section{REFERÊNCIAS}

1. Lima EC, Nascimento GA, Pena MI, Vasconcellos AS, Crepaldi RJQ, Rabelo W, et al. Intervenção coronária percutânea eletiva após fibrinólise: dados do REMAT (Registro Madre Teresa). Rev Bras Cardiol Invasiva. 2011;19(4):373-8.

2. Levine GN, Bates ER, Blankenship JC, Bailey SR, Bittl JA, Cercek B, et al. 2011 ACCF/AHA/SCAI Guideline for Percutaneous Coronary Intervention: executive summary. A Report of the American College of Cardiology Foundation/American Heart Association Task Force on Practice Guidelines and the Society for Cardiovascular Angiography and Interventions. J Am Coll Cardiol. 2011;58(24):2550-83. 